\title{
Laguerre-Gaussian and beamlet array as second generation laser heater profiles
}

\author{
Nikolas Liebster, ${ }^{1,2}$ Jingyi Tang, ${ }^{2}$ Daniel Ratner, ${ }^{2}$ Wei Liu, ${ }^{2}$ Sharon Vetter, ${ }^{2}$ \\ Zhirong Huang, ${ }^{2}$ and Sergio Carbajo, ${ }^{2, *}$ \\ ${ }^{1}$ Rice University, 6100 Main Street, Houston, Texas 77005, USA \\ ${ }^{2}$ Linac Coherent Light Source, SLAC National Accelerator Laboratory, Stanford University, \\ 2575 Sand Hill Road, Menlo Park, California 94025, USA
}

(Received 20 June 2018; published 11 September 2018)

\begin{abstract}
The microbunching instability is known to be detrimental to $x$-ray free electron laser performance. At the Linear Coherent Light Source, the microbunching instability is suppressed with a laser heater, which increases the uncorrelated energy spread of e-beam in the injector. While the current system has been shown to improve x-ray brightness, other laser architectures could further enhance performance. In this study, we model the interaction between a laser and e-beam with arbitrary transverse profiles and examine the effect of various laser designs on the energy distribution of the electrons after the injector and laser heater, as well as their ability to suppress microbunching instability. This simulation incorporates random transverse jitter in order to reproduce physically representative operation of the Linac Coherent Light Source. We compare Gaussian and Laguerre-Gaussian modes, and explore composite beams in the form of an array of Gaussian beamlets. We conclude that the Gaussian laser profile is highly susceptible to e-beam ellipticity and random transverse jitter. The Laguerre-Gaussian profile, a mathematically ideal solution to suppressing microbunching, is less susceptible to these effects and can provide effective suppression even with a distorted e-beam, though performance can be improved by increasing stabilization. The array of beamlets presents a solution that produces consistent and smooth energy distributions with significantly less variance in heating than the Laguerre-Gaussian profile.
\end{abstract}

DOI: 10.1103/PhysRevAccelBeams.21.090701

\section{MOTIVATION AND INTRODUCTION}

Free electron lasers (FEL) are the brightest hard x-ray photon sources in the world, producing femtosecond pulses of photos with wavelengths as low as $1 \AA$ [1-6]. The quality of $\mathrm{x}$-ray brightness and coherence is dependent on the high quality of the e-beam, which is susceptible to the microbunching instability (MBI) [1,7-10]. MBI is caused by initial density modulations in the e-beam which, through bunch compression in the linac, increase the e-beam's slice energy spread beyond FEL tolerances $[1,7]$. This instability can be suppressed by increasing the uncorrelated energy spread of the e-beam with either a superconducting wiggler [11] or a laser heater (LH) [7,11], both of which suppress energy modulations in the e-beam through Landau damping. The LH is a crucial element of the FEL that can significantly improve brightness and allows operation of the FEL in a

\footnotetext{
*Corresponding author. scarbajo@stanford.edu

Published by the American Physical Society under the terms of the Creative Commons Attribution 4.0 International license. Further distribution of this work must maintain attribution to the author(s) and the published article's title, journal citation, and DOI.
}

variety of modes, in particular self-seeding or harmonic lasing [12-15]. At the Linac Coherent Light Source (LCLS), the LH increases the initial energy spread of the e-beam by an order of magnitude without exceeding FEL tolerances. Heating occurs along a small undulator within a magnetic chicane at an e-beam energy of $135 \mathrm{MeV}$ [1].

The e-beam energy distribution after the LH is highly dependent on the transverse profile of the LH beam, making investigations of transverse LH intensity distributions particularly relevant [11]. The current LH at LCLS employs a Gaussian transverse beam distribution matched to the transverse e-beam shape [1]. However, recent theoretical studies have investigated Laguerre-Gaussian 01 (LG01) and other unconventional transverse beam distributions that may provide better suppression of microbunching; the LG01 mode, for instance, provides a mathematically ideal solution to suppressing MBI under controlled conditions [16]. Under ideal laser and e-beam conditions, the suppression of microbunching using a LG01 beam profile has been shown to be significantly better than the matched Gaussian laser [16]. For ideal suppression, the rms laser spot size can be up to 4 or 5 times the rms e-beam size, thereby exhibiting more modematching flexibility than in the Gaussian case.

Performance of any given LH varies greatly depending on practical parameters of the accelerator, such as e-beam 
distribution mismatch, spatial jitter or smearing effects, and no previous study has examined the use of these various transverse LH profiles with respect to these practical considerations. As an example of these effects, the e-beam transverse profile at LCLS is variable and typically elliptical. Additionally, acoustic and thermal noise can cause both pointing and transverse offset jitter in the LH pointing, thus compromising LH and e-beam spatial overlap from shot to shot.

In this study, we examine the effect of random transverse jitter and elliptical e-beam profiles on energy distributions induced by the current $\mathrm{LH}$ to resemble routine beam operation at LCLS. We explore alternative LH designs that outperform state-of-the-art LH architectures under these considerations based on either LG01 beams or a Gaussian beamlet array (BA). By using a computational model that simulates the interaction between arbitrary transverse laser and e-beam distributions, we examine various methods to generate Gaussian-like e-beam energy distributions. This is because MBI is suppressed more when this distribution is more Gaussian due to more effective Landau damping [11,17]. We also compare the quantified MBI suppression in both LG01 and BA approaches, and discuss the tradeoffs between both designs. We conclude that Gaussian beams have practical limitations due to beam overlap and alignment instabilities compared to either a LG01-mode laser distribution with enhanced stabilization or a discretized array of beamlets.

\section{METHODS}

The following equation describes the induced energy modulation from an arbitrary laser profile interacting with an e-beam, assuming that the small changes in size between the e-beam and laser due to the resonant interaction are negligible [11]:

$$
\begin{aligned}
\delta_{L}(x, y)= & \sqrt{\frac{P_{L}}{P_{0}}} \frac{K L_{u}}{\gamma_{0} \sigma_{r}} \\
& \times\left[J_{0}\left(\frac{K^{2}}{4+2 K^{2}}\right)-J_{1}\left(\frac{K^{2}}{4+2 K^{2}}\right)\right] h(x, y) .
\end{aligned}
$$

Here, $P_{L}$ is the peak laser power, $P_{0}=I_{A} * m c^{2} / e \sim$ 8.7 GW, $K$ is the undulator strength parameter, $L_{u}$ is the undulator length, $\gamma_{0}$ is the relativistic factor, $\sigma_{r}$ is the rms laser spot size, $J_{0}$ and $J_{1}$ are the Bessel functions of the first kind, and $h(x, y)$ is a normalized, arbitrary transverse laser intensity profile with a center at the origin $(0,0)$. Because offsets between the e-beam and laser field are relative, the laser is defined as centered on the origin while offsets are incorporated into the e-beam distribution, $g(x, y)$. The initial energy spread of the e-beam is assumed to be Gaussian in shape due to the low energy spread and noisy nature of the process in which electrons are generated.
Thus, the new electron distribution in energy and space after the electron-laser interaction is described by

$$
\begin{aligned}
f_{0}\left(z_{0}, \delta_{0}, r\right)= & \frac{I_{0}}{e c \sqrt{2 \pi} \sigma_{\gamma_{0}}} \\
& \times \exp \left[-\frac{\left(\delta_{0}-\delta_{L}(r) \sin k_{L} z_{0}\right)^{2}}{2 \sigma_{\gamma_{0}}^{2}}\right] g(x, y),
\end{aligned}
$$

where $I_{0}$ is the maximum instantaneous e-beam current, $\sigma_{\gamma_{0}}$ is the initial e-beam rms energy spread, $k_{L}$ is the wave number of the laser, $\delta_{0}$ is the modification of the relativistic constant of an electron due to the interaction, $g(x, y)$ is the initial transverse spatial distribution of the e-beam, and $z_{0}$ is the longitudinal coordinate with $z_{0}=0$ defined as the e-beam head. Typically, $g(x, y)$ is defined as the twodimensional Gaussian function, which incorporates translational and angular offsets. We use the origin of the laser intensity distribution as the universal origin and incorporate offsets by manipulating $g(x, y)$ as follows:

$$
\begin{aligned}
g(x, y)= & B \cdot \exp \left[-\left(a\left(x-x_{0}\right)^{2}+2 b\left(x-x_{0}\right)\left(y-y_{0}\right)\right.\right. \\
& \left.\left.+c\left(y-y_{0}\right)^{2}\right)\right],
\end{aligned}
$$

where $B$ is a normalization constant, $x_{0}$ and $y_{0}$ are the transverse offsets in $x$ and $y$, respectively, with the matrix

$$
\left[\begin{array}{ll}
a & b \\
b & c
\end{array}\right]
$$

being positive definite with coefficients $(a, b$, and $c$ ) that are related to the angular offset $(\theta)$ and transverse beam spot sizes $\left(\sigma_{x}, \sigma_{x}\right)$ as follows:

$$
\begin{aligned}
& a=\frac{\cos ^{2}(\theta)}{2 \sigma_{x}^{2}}+\frac{\sin ^{2}(\theta)}{2 \sigma_{y}^{2}} \\
& b=-\frac{\sin (2 \theta)}{4 \sigma_{x}^{2}}+\frac{\sin (2 \theta)}{4 \sigma_{y}^{2}} \\
& c=\frac{\sin ^{2}(\theta)}{2 \sigma_{x}^{2}}+\frac{\cos ^{2}(\theta)}{2 \sigma_{y}^{2}} .
\end{aligned}
$$

When (2) is integrated over all space, a probability density function is generated, as a function of the difference from the nominal beam energy:

$$
V\left(\delta_{0}\right)=2 \pi \int r d r \int d z_{0} f_{0}\left(z_{0}, \delta_{0}, r\right) .
$$

To quantify MBI suppression, we look at microbunching gain, defined in [11] as 


$$
G=\left|\frac{b_{f}}{b_{0}}\right|=\frac{I_{0}}{\gamma I_{A}}\left|k_{f} R_{56} \int_{0}^{L} d s \frac{4 \pi Z\left(k_{f}\right)}{Z_{0}}\right| S_{L}
$$

where $b_{0}$ and $b_{f}$ are the initial and final bunching factors, and $k_{f}$ is the compressed modulation wave number. The suppression factor, $S_{L}$, is defined as the Fourier transform of the energy distribution,

$$
S_{L}\left(k_{f}\right)=\int d \delta_{0} V\left(\delta_{0}\right) e^{-i k_{f} R_{56} \delta_{0}} .
$$

The longitudinal space charge impedance function is defined as

$$
Z(k, s)=\frac{i Z_{0}}{\pi k r_{b}^{2}}\left[1-\frac{k r_{b}}{\gamma} K_{1}\left(\frac{k r_{b}}{\gamma}\right)\right] .
$$

The current variance is proportional to $\int_{0}^{\infty} d k_{0} G^{2}\left(k_{0}\right)$ [11], and thus we define

$$
I=\int_{0}^{\infty} d k_{0} G^{2}\left(k_{0}\right)
$$

where a lower $I$ indicates less current variance and therefore more effective MBI suppression. The parameter is not absolute, as it measures only suppression of MBI and not FEL performance. Heating the beam infinitely would fully suppress MBI and result in a low $I$ but would be detrimental to FEL performance. Thus, we consider $I$, the shape of $V\left(\delta_{0}\right)$ and $|S|$, and the stability of the energy spread when incorporating offset, tilt, and nonzero eccentricity when determining the effectiveness of heating of various LH designs.

The simulation, developed in MATLAB, uses (1)-(3) to generate the modified e-beam energy distribution after the laser-electron interaction by modeling transverse interactions between the laser and the electron. When using Gaussian LH profiles, we define the intensity distribution as

$$
h(r)=A \cdot e^{-r^{2} / 2 \sigma_{r}^{2}},
$$

and the LG01 profile as

$$
h(r)=A \cdot r \cdot e^{-r^{2} / 2 \sigma_{r}^{2}}
$$

Here, $r$ is the transverse radial cylindrical coordinate due to circular symmetry, and the coefficient $A$ is a normalization constant that sets the maximum of the intensity distribution to 1 . The BA intensity distribution is defined as a series of small, transversely and longitudinally copropagating superimposed Gaussian intensity distributions.

The simulation does not account for longitudinal effects such as chirp or space charge effects due to the high energy of the electrons at the end of the LCLS photoinjector $(135 \mathrm{MeV})$; at such high energies, these effects
TABLE I. Relevant LCLS parameter values.

\begin{tabular}{lcc}
\hline \hline Parameter & Symbol & Value \\
\hline Electron energy & $\gamma_{0} m c^{2}$ & $135 \mathrm{MeV}$ \\
Undulator strength parameter & $K$ & 1.56 \\
Undulator length & $L_{u}$ & $0.5 \mathrm{~m}$ \\
Initial peak current & $I_{0}$ & $30 \mathrm{~A}$ \\
Initial energy spread & $\sigma_{\gamma_{0}}$ & 0.006 \\
Momentum compaction & $R_{56}$ & $45 \mathrm{~mm}$ \\
Free space impedance & $Z_{0}$ & $377 \Omega$ \\
Radius of cross section for uniform & $r_{b}$ & $300 \mu \mathrm{m}$ \\
$\quad$ electron distribution & $\sigma_{x} / \sigma_{y}$ & $200 \mu \mathrm{m} / 200 \mu \mathrm{m}$ \\
Ideal transverse e-beam sizes & $\sigma_{x} / \sigma_{y}$ & $115 \mu \mathrm{m} / 345 \mu \mathrm{m}$ \\
Distorted transverse e-beam sizes & $L$ & $12 \mathrm{~m}$ \\
Linac 1 length & $\delta_{x} / \delta_{y}$ & $140 \mu \mathrm{m}$ \\
Standard deviation of transverse & \multicolumn{2}{c}{$\sim$} \\
$\quad$ offset & $\delta_{E}$ & $\sim$ \\
\hline \hline
\end{tabular}

are considered negligible, especially in comparison to transverse effects.

At LCLS, distributions with an energy spread, $\delta_{E}$, of around $20 \mathrm{keV}$ rms produce the highest $\mathrm{x}$-ray intensity [1]. MBI suppression results from the simulation, however, vary depending on the use of rms or FWHM energy spread definitions. The rms definition rewards beams that have small tails, while the FWHM metric more clearly illustrates beam stability. These differences are highlighted when analyzing the effectiveness of varying beam profiles at suppressing MBI. Thus, for each profile we present results for distributions that are optimized for a $20 \mathrm{keV}$ spread in both rms and FWHM. Unless specified, parameters for figures are chosen to approximately reflect $\delta_{E}=$ $20 \mathrm{keV}$ rms.

The parameters and values used in our system, specified in Table I, are based on recent data from and current performance of LCLS. Though the results presented in this paper use LCLS-specific accelerator parameters, the qualitative conclusions are applicable to other FELs, and the proposed LH systems can be generalized to other operation regimes.

When the LH at LCLS was first proposed, a Gaussian laser with a transverse rms width, $\sigma_{r}$, of $\sqrt{2} \sigma_{x}$ was assumed [11]. In practice, both laser and e-beam intensity distributions are aberrated, making it difficult to achieve adequate mode matching. The laser pulses are generated from a Ti: sapphire amplifier system at a wavelength of $760 \mathrm{~nm}$, and pulse energies at interaction usually range from 5-20 $\mu \mathrm{J}$ with a pulse duration of around 10-20 ps. Internal data [18] shows that the laser field and e-beam centers have independent shot-to-shot offsets with standard deviations of around $100 \mu \mathrm{m}$ rms during routine operation. Using error propagation for uncorrelated error, we define the standard deviation of randomly generated offset between the laser and e-beam centers in the $\mathrm{x}$ and $\mathrm{y}$ directions, $\delta_{x}$ and $\delta_{y}$, 
as $140 \mu \mathrm{m}$. Operational data also shows that the e-beam ellipticity can have an aspect ratio of up to 3 .

In this study we add both transverse offset jitter and ellipticity to the beams to investigate their effect on heating and MBI suppression, as depicted in Fig. 1. The profile of the e-beam was made to be elliptical with an aspect ratio of 3 . Offsets and pointing angles were generated with random Gaussian noise. Offsets were generated independently in $\mathrm{x}$ and y directions, with standard deviations of $140 \mu \mathrm{m}$ in each direction, while standard deviation for the angle was 20 degrees, to accurately represent routine LCLS operations.

\section{GAUSSIAN AND LG01 PROFILE RESULTS}

We benchmark our simulation tool by sweeping peak power and laser beam waist to ensure that energy distributions from published results are reproducible. The shapes produced for the mode-matched Gaussian laser intensity distribution as well as an oversized laser field, shown in Fig. 2, match previous theoretical results [11]. A transversely Gaussian LH beam significantly larger than the mode-matched ratio is in principle unable to effectively suppress MBI regardless of its power. This is because, in essence, an oversized Gaussian laser distribution is equivalent to a flattop transverse distribution, which inevitably results in the double-horn heated energy distribution shown in Fig. 2. This high sensitivity to mode matching is specific to transversely Gaussian LH beams and has been studied in detail in [11]. Our results also show that a $\sigma_{r} \sim 200 \sqrt{2} \mu \mathrm{m}$ with a circular e-beam of transverse rms size of $200 \mu \mathrm{m}$ yields the most effective MBI suppression, with $I_{\mathrm{rms}} \sim$ $1800 \mathrm{~m}^{-1}[11,16]$. As we will describe in this section, the laser power level required for the LG01 mode to produce the same e-beam energy spread is significantly higher than that for the Gaussian laser. This is primarily because only the inner intensity of the LG01 mode is used effectively for heating. Thus, the size of either beam is chosen based on the optimal mode-matching condition [11], and power is set such that energy spreads after the heater are comparable.

Beyond benchmarking our simulation, the three $V\left(\delta_{0}\right)$ shown in Fig. 2 serve as a reference for energy distributions produced by other laser distributions at varying jitter. The ideal case for a given e-beam and laser intensity distribution, i.e., the heating resulting from no offset between the laser and e-beam centers, is plotted in red. The two nonideal shapes in Fig. 2 represent heating with a constant laser intensity distribution, i.e., a Gaussian distribution with a $\sigma_{r} \gg \sigma_{x}$, as well as one with little to no heating. In the oversized case, two peaks form at the maximum energy modulation, $\delta_{0} \sim \pm \delta_{L}(0)$, indicating that the e-beam is energetically split into two groups each with a small energy spread. It is important to note that this occurs for an oversized laser intensity distribution, not an overpowered one, as increasing power typically increases $\delta_{E}$ without significantly impacting the shape of $V\left(\delta_{0}\right)$. In the underheated case, the original Gaussian energy distribution with an rms width of $3 \mathrm{keV}$ of the e-beam is recovered. The over- and underheated shapes of these curves appear consistently across $\left(V \delta_{0}\right)$, particularly when incorporating jitter.

As stated previously, when quantifying MBI suppression, we use two definitions of energy spread, rms and FWHM. First, laser parameters are adjusted to yield $\delta_{E}=$ $20 \mathrm{keV}$ using either definition while the e-beam and laser profiles are centered with no offset. Jitter is then incorporated with no e-beam ellipticity, and $I$ and $\delta_{E}$ values are averaged over 100 iterations. These results are summarized in Table II. For a Gaussian LH, the simulation indicates that $P_{L}=0.4 \mathrm{MW}$ induces an rms energy spread of $\sim 20 \mathrm{keV}$, while $P_{L}=0.05 \mathrm{MW}$ induces a FWHM spread of the same magnitude.

Incorporating transverse offset jitter alone reveals shortcomings of the Gaussian laser profile. As shown in the overlaid distributions in Fig. 3(a), the energy redistributions for the Gaussian profile deviate significantly from their ideal mode-matched distributions (in red). Standard deviations off of mean $I$ values for both rms and FWHM cases are at least as large as the values themselves. It should be noted that $I$ is positive definite, and for the Gaussian case heating can only be worsened with increased offset. Offset between the e-beam and the Gaussian laser causes a significant decrease in heating, as expected. The thin, sharp central peak that emerges in Fig. 3(a) is the result of lesser heating because of lack of overlap, and partially represents the initial bunch energy distribution.
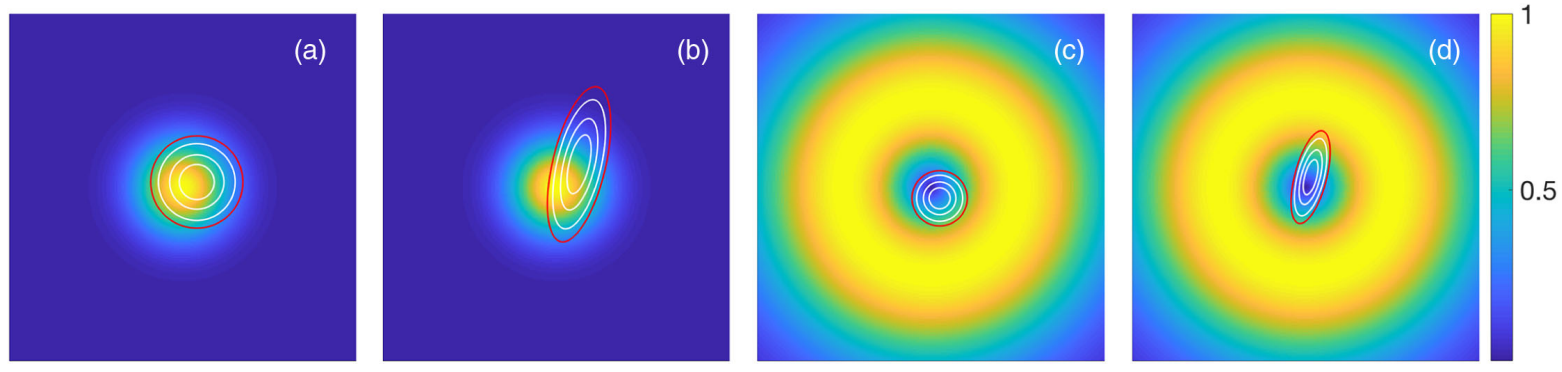

FIG. 1. LH and e-beam intensity distributions. The heat-map represents the laser intensity while the contour plot represents the probability distribution of the e-beam. The red line is the $e^{-1}$ mark. 


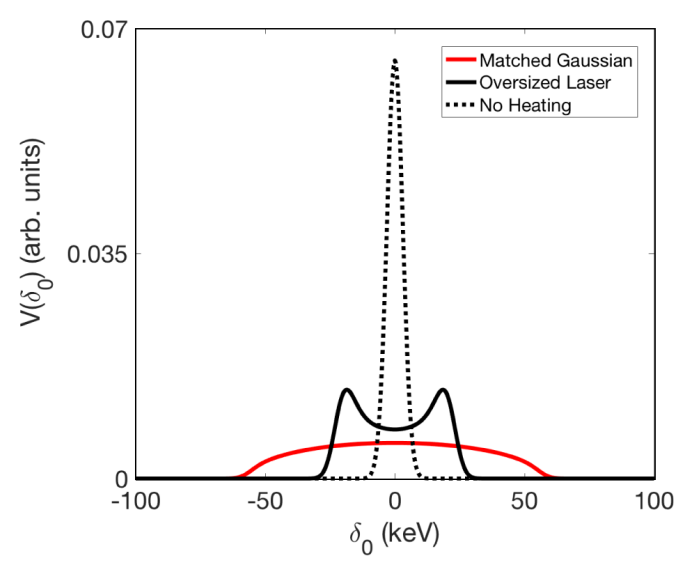

FIG. 2. Energy distributions due to a Gaussian laser profile. When the laser becomes oversized, the e-beam is split into two unheated groups at $\pm \delta_{L}(0)$, the maximum energy modulation. The matched Gaussian distribution is the theoretically predicted energy distribution of the e-beam after heating with the current LH.

Once e-beam ellipticity is incorporated, as shown in 3(b), it is no longer possible to generate a Gaussian-like e-beam energy distributions even without offset or tilt, and even energy distributions for perfectly centered beams have a significant pedestal around the central peak. The solution, as is currently in practice at LCLS, is to match the laser profile to the ellipticity of the e-beam so as to recover the matched-Gaussian case, which demands additional mode matching and alignment procedures.

The LG01 mode, in contrast, can generate a perfectly Gaussian e-beam energy distribution in ideal, mode-matched conditions. The simulations approach the mathematically perfect solution when the laser spot size is at least 5 times larger than $\sigma_{r}$, assuming cylindrical symmetry. For our testing, we use $\sigma_{r}=1.3 \mathrm{~mm}$, with $P_{L}=20 \mathrm{MW}$ for an rms energy spread of about $20 \mathrm{keV}$ and $P_{L}=3 \mathrm{MW}$ for the same spread in FWHM.

The most significant drawback of the LG01 method is its higher power consumption than in the Gaussian case. Its power consumption is at least 1 order of magnitude higher than the Gaussian laser's, if not more.

The distributions generated by the LG01 mode when incorporating only offset jitter also show deviations from the ideal mode-matched case, with a higher density of distributions close to the ideal mode-matched case. When incorporating a nonzero eccentricity, the distributions vary even more widely and inconsistently, as shown in Fig. 3(c). Due to random jitter, the e-beam may occasionally overlap with the intensity minimum in the center of the beam, and other times lie along the intensity maximum at the ring. However, because of the larger size of the LG01 laser, this happens relatively infrequently and could be avoided if the beams were stabilized to reduce jitter. In our case study at LCLS, an offset jitter below $80 \mu \mathrm{m}$ rms would provide consistent and stable heating.

Using the rms spread measurement, the LG01 is highly resilient to both offset and e-beam distortion. Even without added stabilization the LG01 represents significant improvement to the Gaussian laser profile. For the ideal e-beam, we calculate mean $I_{\text {rms }}=800 \mathrm{~m}^{-1}$ with standard deviation of $400 \mathrm{~m}^{-1}$, and suppression factor shapes remain relatively Gaussian in shape. With an elliptical e-beam, we calculate a similar gain, with $I_{\text {rms }}=800 \mathrm{~m}^{-1}$ and a standard deviation of $300 \mathrm{~m}^{-1}$, with suppression factors varying slightly more significantly. Both of these cases show significant improvement from the Gaussian case, with $I$ values of up to 37 times lower than the Gaussian profile for rms energy spreads, and 1 order of magnitude better for FWHM spreads.

Optimizing curves using the FWHM measure results in worse suppression for the LG01 beam, as well as greater variance when incorporating jitter. We find $I_{\mathrm{FWHM}}=$ $4 \times 10^{4} \mathrm{~m}^{-1}$ for the circular e-beam case, with a standard deviation around half of the mean value. For the elliptical e-beam, we find $I_{\mathrm{FWHM}}=3 \times 10^{4} \mathrm{~m}^{-1}$ with a standard deviation of $3 \times 10^{4} \mathrm{~m}^{-1}$. We see that comparatively the ellipticity of the e-beam has little effect on the suppression. The variance for both cases, however, is significant, indicating that the displacement of the e-beam in relation to the laser field may have a significant impact on consistency of heating.

One possible solution to decreasing the variance in energy distributions is by stabilizing the laser and e-beam. This could be achieved in the form of waveguide beam propagation for the laser heater as opposed to free-space propagation, for instance, and by means of apertures and beam stabilizers for the e-beam. Another alternative is

TABLE II. Energy spread and $I$ values for Gaussian and LG01 intensity distributions for both rms and FWHM optimization.

\begin{tabular}{|c|c|c|c|c|c|c|}
\hline Laser Profile & Energy spread & e-beam ellipticity & $P_{L}(\mathrm{MW})$ & $\delta_{E}(\mathrm{keV})$ & $I\left(\mathrm{~m}^{-1}\right)$ & stdev of $I\left(\mathrm{~m}^{-1}\right)$ \\
\hline \multirow[t]{4}{*}{ Gaussian } & rms & $1: 1$ & 0.4 & $20 \pm 2$ & $0.7 \times 10^{4}$ & $2 \times 10^{4}$ \\
\hline & & $1: 3$ & 0.4 & $18 \pm 2$ & $3 \times 10^{4}$ & $3 \times 10^{4}$ \\
\hline & FWHM & $1: 1$ & 0.05 & $20 \pm 4$ & $0.9 \times 10^{4}$ & $1 \times 10^{4}$ \\
\hline & & $1: 3$ & 0.06 & $16 \pm 3$ & $1.5 \times 10^{4}$ & $1.5 \times 10^{4}$ \\
\hline \multirow[t]{4}{*}{ LG01 } & $\mathrm{rms}$ & $1: 1$ & 20 & $20 \pm 3$ & 800 & 400 \\
\hline & & $1: 3$ & 18 & $21.5 \pm 1.6$ & 800 & 300 \\
\hline & FWHM & $1: 1$ & 3 & $21 \pm 4$ & $4 \times 10^{4}$ & $2 \times 10^{4}$ \\
\hline & & $1: 3$ & 3 & $23 \pm 5$ & $3 \times 10^{4}$ & $1 \times 10^{4}$ \\
\hline
\end{tabular}



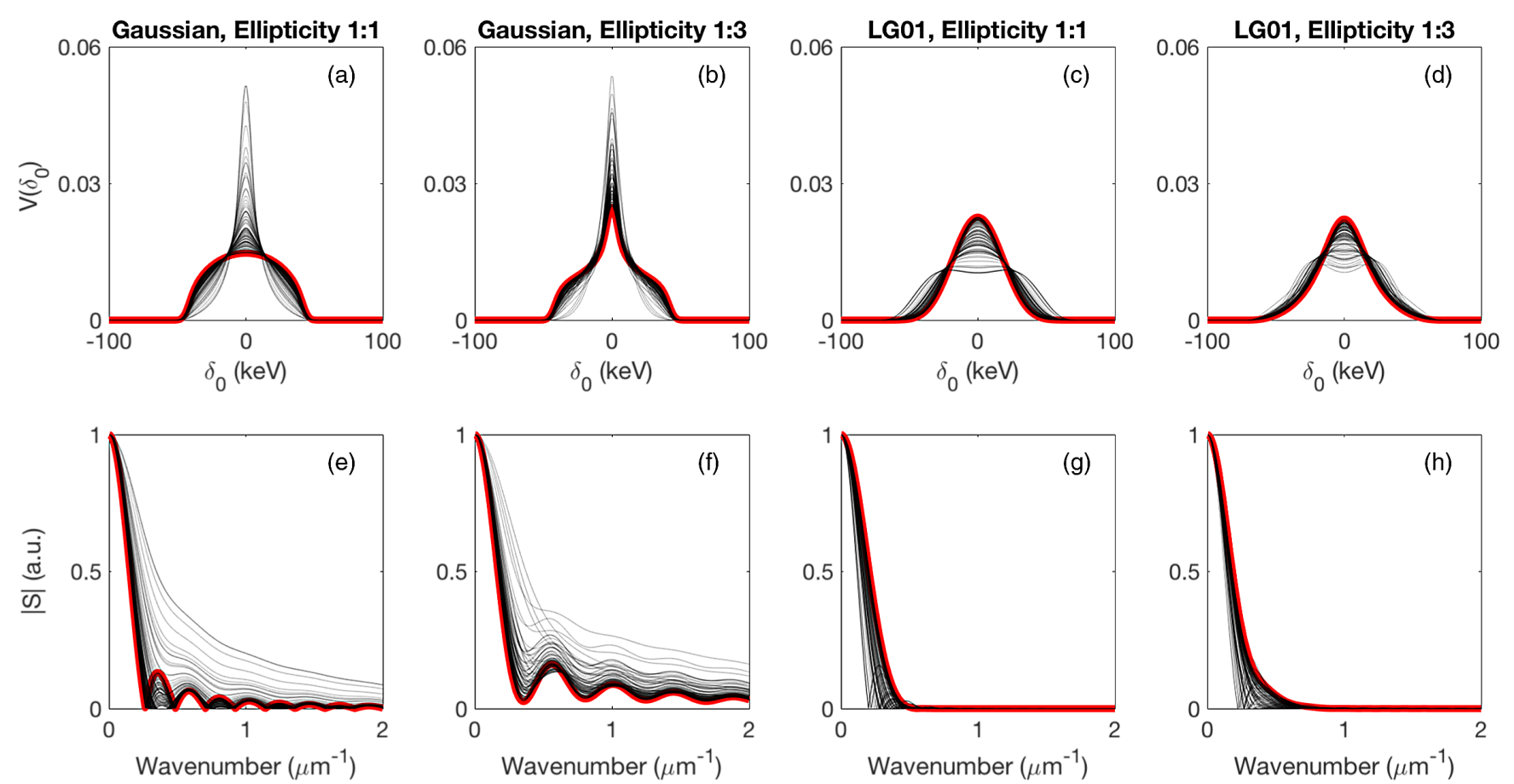

FIG. 3. Energy distributions and suppression factors for Gaussian and LG01 beams, with and without e-beam ellipticity. Parameters are set for $20 \mathrm{keV} \mathrm{rms} \mathrm{energy} \mathrm{spread.} \mathrm{The} \mathrm{red} \mathrm{line} \mathrm{is} \mathrm{the} \mathrm{distribution} \mathrm{generated} \mathrm{when} \mathrm{offset} \mathrm{and} \mathrm{pointing} \mathrm{angle} \mathrm{are} \mathrm{zero.} \mathrm{Each} \mathrm{of} \mathrm{the} 100$ superimposed distributions is generated with a random offset with identical laser and e-beam parameters.

creating a laser field that exhibits on a shot-to-shot basis the same nominal heating independent of jitter and mode matching. This motivates the study of the beamlet array as an alternative to LG01 and Gaussian LH distributions, as discussed in the following section.

\section{BEAMLET ARRAY}

In the face of high stabilization and beam-tuning requirements of the Gaussian beams, we propose a third LH candidate, a discretized array of beamlets. Conceptually, a BA serves to create highly discretized sources of localized heating points. This design overcomes the limitations of traditional profiles because heating is extremely robust to instabilities caused by beam size, tilt angle, and transverse offset jitter. It can offer Cartesian or cylindrical symmetry over a larger surface area such that these transverse effects have a reduced impact on energy distributions after the LH.

Previous work has investigated the effectiveness of the $\mathrm{BA}$ in ideal cases and designed a mathematically ideal laser distribution with non-Gaussian beamlets that results in perfect e-beam heating [16]. Due to the difficulty of propagating non-Gaussian beamlets through the length of the LH undulator, we examine the use of a BA constructed using simple Gaussian beamlets as an initial exploration into the feasibility and characteristics of this design. Due to the difficulty of propagating non-Gaussian beamlets through the length of the LH undulator, we examine the use of a BA constructed using simple Gaussian beamlets as an initial exploration into the feasibility and characteristics of this design before delving into the one first proposed in [16].

The Gaussian BA design imposes dramatically looser requirements for e-beam ellipticity, stabilized pointing, or mode matching. That is, the BA allows the e-beam to see similar heating regardless of where on the laser profile it lands, provided that the BA is sufficiently discretized, as we will study in this section. This rationale is verified using both circular and elliptical e-beams including the same transverse offset jitter and tilt values as those in Sec. III. Under these conditions, the BA is able to consistently sustain a Lorentzian-like e-beam energy distribution with and without jitter and eccentricity.

\section{A. Parameter optimization}

To find a design which is impervious to offsets and tilts that results in a modified energy distribution that best matches a Gaussian-like linewidth with $20 \mathrm{keV}$ rms energy spread, we begin by sweeping peak power, defined as the sum of individual beamlet peak powers, as well as beamlet rms spot size and beamlet discretization. Here too, the total amount of power required by the BA is significantly higher than that for the Gaussian distribution. In this case, the higher power levels are associated to the fact that only a subset of the beamlets in the entire array are effectively interacting with the electron bunch at any given event. Discretization is swept by varying interbeamlet spacing and defining spot size at a fixed fraction of this distance. 

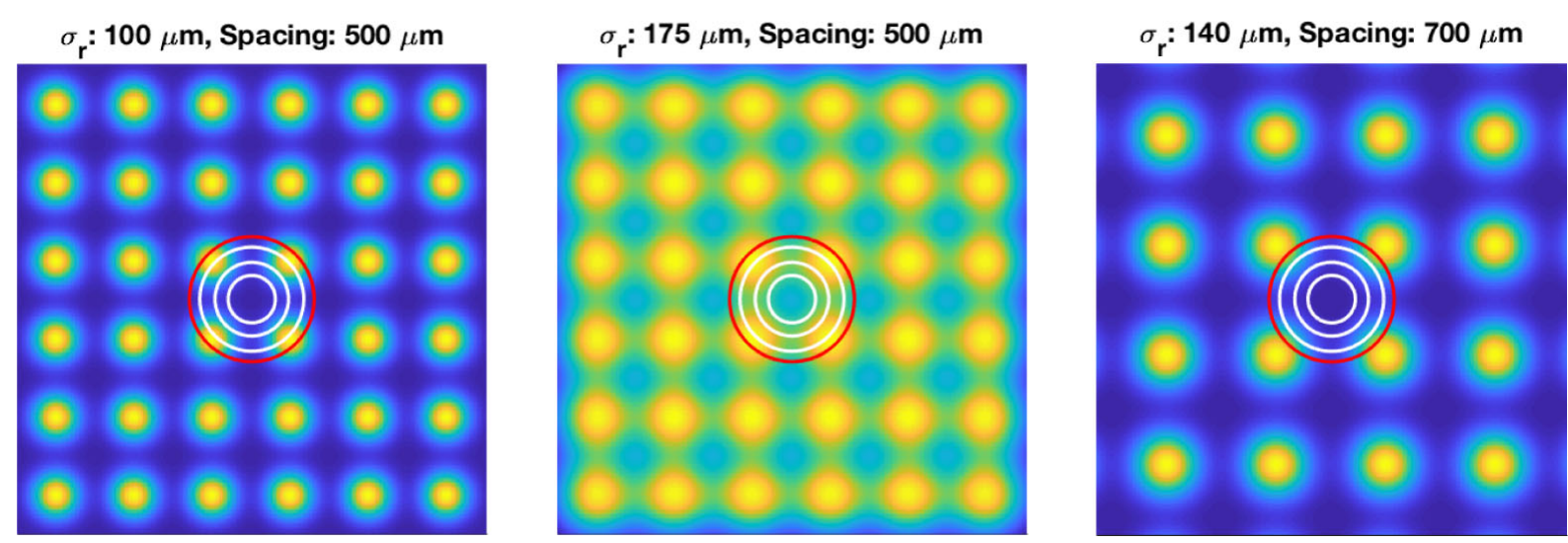

FIG. 4. Illustration of BA profile with various fill and spacing factors. The left profile shows the ideal parameters determined by sweeping. The center panel shows an increased fill factor, while the right shows larger beamlets.

Beamlet spot size is swept by keeping the interbeamlet spacing constant while changing beamlet rms width, changing the fill factor of the profile. By sweeping these two spatial parameters as well as peak power, we investigate all possible effects on the energy distribution of the electrons after heating such that the final energy spreads are comparable to the previous two cases.

We begin a parametric study by sweeping peak power and beamlet waists for a transversely circular e-beam. The e-beam has a $\sigma_{r}$ of $200 \mu \mathrm{m}$, as was used for circular testing with Gaussian and LG01 LH profiles in Sec. III. For parameter sweeps, we define the e-beam as transversely and longitudinally centered on the BA when the center of the e-beam distribution aligns with an intensity minimum of the LH profile, depicted in Fig. 4.

Preliminary testing shows that $500 \mu \mathrm{m}$ beamlet spacing, i.e., approximately a minimum of four overlapped beamlets per e-beam, is a reasonable discretization, and this parameter is used throughout fill factor and power sweeping. A $6 \times 6$ pattern is used, resulting in a $3 \mathrm{~mm} \times 3 \mathrm{~mm}$ square BA distribution. The powers swept range from 1.5 to $50 \mathrm{MW}$ in $7 \mathrm{MW}$ intervals. This sweeps the range of reasonable powers, centered at a level that is commensurate with our previous Gaussian and LG01 study. For each power, a laser waist, defined as $\sigma_{r}$ using (10), is also swept over a range from 25 to $250 \mu \mathrm{m}$ in $25 \mu \mathrm{m}$ intervals. These spot sizes span designs with almost no fill to a practically flattopped laser profile.

We note that sustaining these small beamlets over the current undulator length is difficult due to limited Rayleigh ranges. For simplicity of comparison to laser intensity distributions from the previous section, we have purposefully decided to maintain the same dimensions as previously for comparison purposes. In the future, propagation limitations could be addressed by either scaling dimensions and power proportionately to meet the current LH undulator design or by redesigning the $\mathrm{LH}$ undulator to be shorter.
The shapes of resulting distributions are displayed in Fig. 5. As expected, increasing fill factor and peak power also increases the induced energy spread of the distribution, and eventually leads to a double-horn distribution, analogous to a single Gaussian beam in the extreme case of very large beamlet size as shown in Fig. 2. Decreasing spot size results in little to no heating, with shapes resembling the initial energy distribution of the electrons before the LH. For a circular e-beam with a $\sigma_{r}$ of $200 \mu \mathrm{m}$ and a beamlet spacing of $500 \mu \mathrm{m}$, a practical beamlet waist is determined to be around $100 \mu \mathrm{m}$.

Once an adequate fill factor is determined, we can proceed to sweep beamlet spacing to ensure that the chosen level of discretization is appropriate. Figure 6 shows the energy distributions resulting from a power and beamlet spacing sweep, where the beamlet waist size is kept constant in reference to the distance between intensity maximums. Using the same powers as in the fill sweep, beamlets with spacings of $100 \mu \mathrm{m}$ up to $1000 \mu \mathrm{m}$ in $100 \mu \mathrm{m}$ increments are tested. Beamlet spot size is set at 0.2 times beam spacing, to be consistent with the reasonable fill determined above. We assume the beamlets to be incoherent with respect to one another, therefore neglecting interference between beamlets.

Figure 6 evidences that the shape of energy distributions changes primarily in the spread width rather than line shape, until discretization is too large. As beamlet spacing increases, the troughs in which the e-beam is found become larger, causing less heating, as shown in Fig. 4. If random offsets are incorporated in cases of large beamlet spacing, a larger disparity in heating between the intensity troughs and peaks is expected depending on which portion of the profile the e-beam lands on, increasing variability in energy spreads. Under this logic, for a circular transverse e-beam profile with a radius of $200 \mu \mathrm{m}$, spacing should not be much larger than $600 \mu \mathrm{m}$. In this geometric configuration, a total peak power of 15-30 MW provides an rms energy spread of about $20 \mathrm{keV}$. 

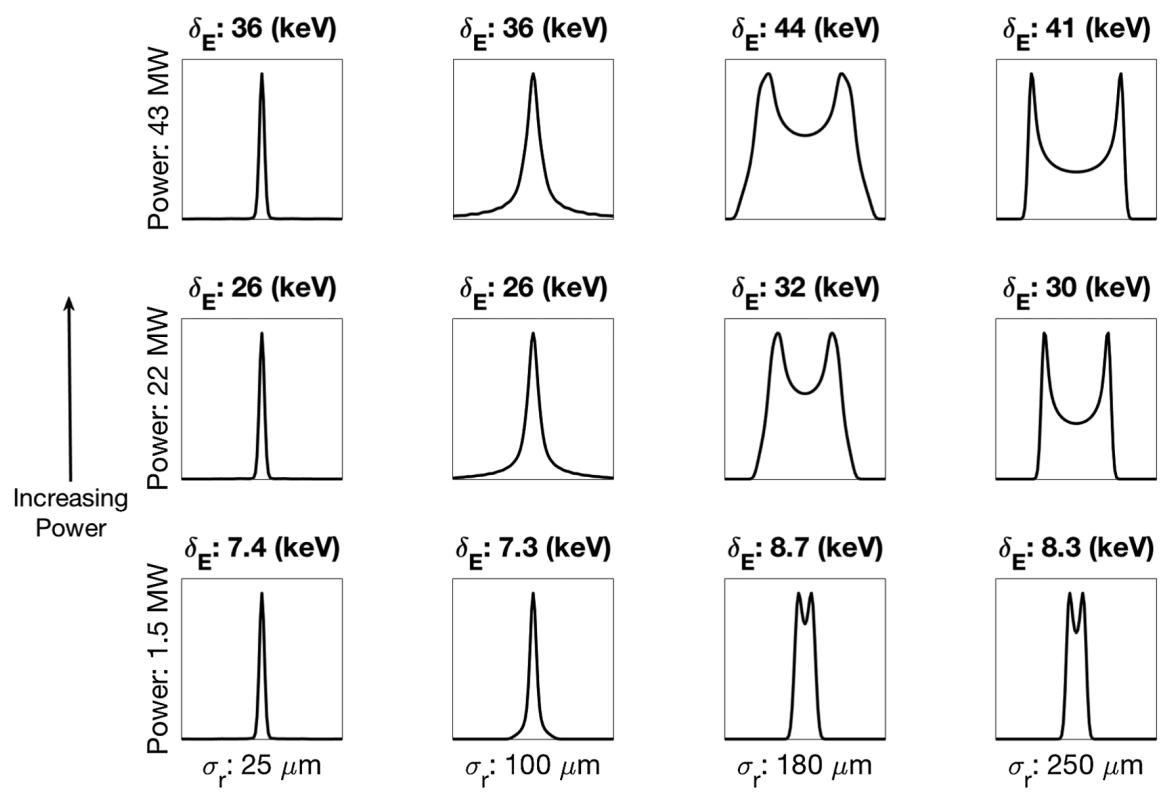

Increasing Fill

FIG. 5. Energy distributions and their averaged rms energy spread $\left(\delta_{E}\right)$ over a sweep of beamlet waist with a fixed distance between beamlet centers, defined as fill factor. Large $\delta_{E}$ values for thin distributions are due to small aberrations at high $\delta_{0}$ due to large laser power but small probability of interaction. Plots are normalized with respect to maximum value.
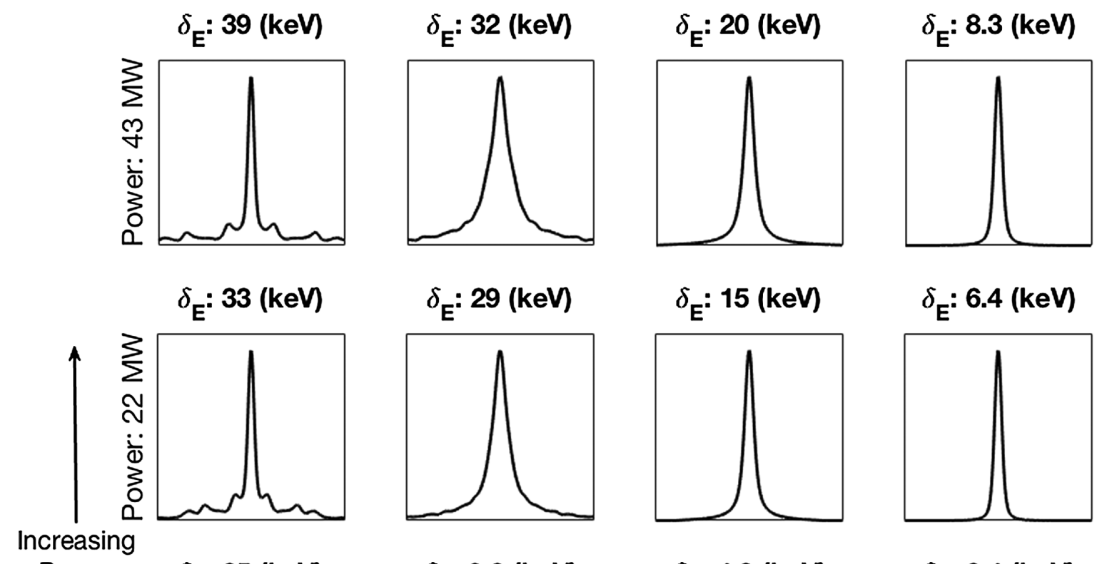

$\delta_{\mathrm{E}}: 6.4(\mathrm{keV})$
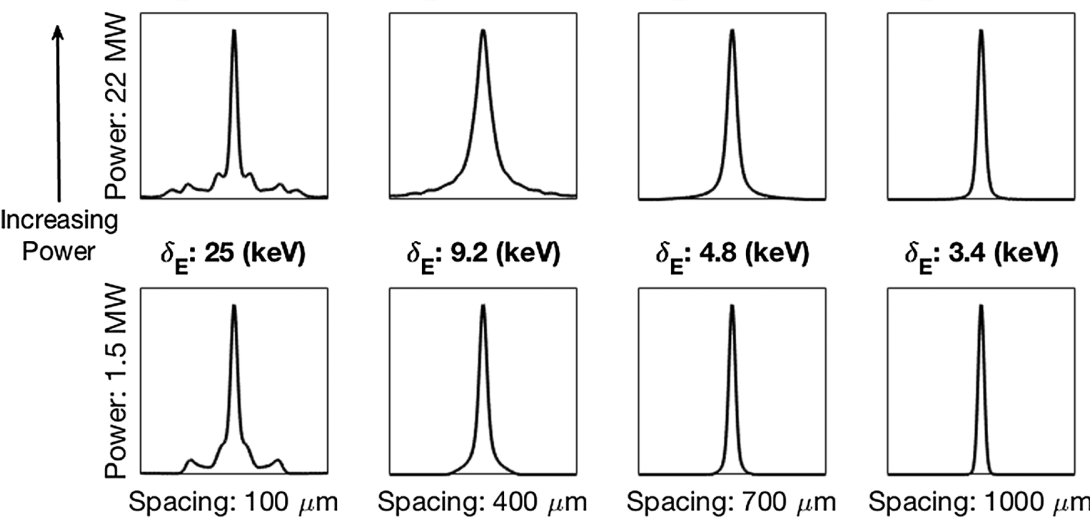

Increasing Discretization

Spacing: $700 \mu \mathrm{m} \quad$ Spacing: $1000 \mu \mathrm{m}$

FIG. 6. Energy distributions and their averaged rms energy spread $\left(\delta_{E}\right)$ over a sweep of spacing between beamlet centers with $\sigma_{r}$ set at $1 / 5$ of spacing. The change in interbeam spacing effectively sweeps the discretization of the beam. Ripples in curves intensify due to small beamlet with increasing power. Plots are normalized with respect to maximum value.

An adequate adjustment of the beamlet size and spacing proves to allow for energy spread tuning without additional mode-matching considerations, thereby introducing a practical feature that is highly insensitive to variations of the e-beam position and ellipticity relative to the BA. For the LCLS-specific parameters, a beamlet spot size of $100 \mu \mathrm{m}$ and beamlet spacing of $500 \mu \mathrm{m}$ are chosen. Peak power of $12 \mathrm{MW}$ induces an rms energy spread 

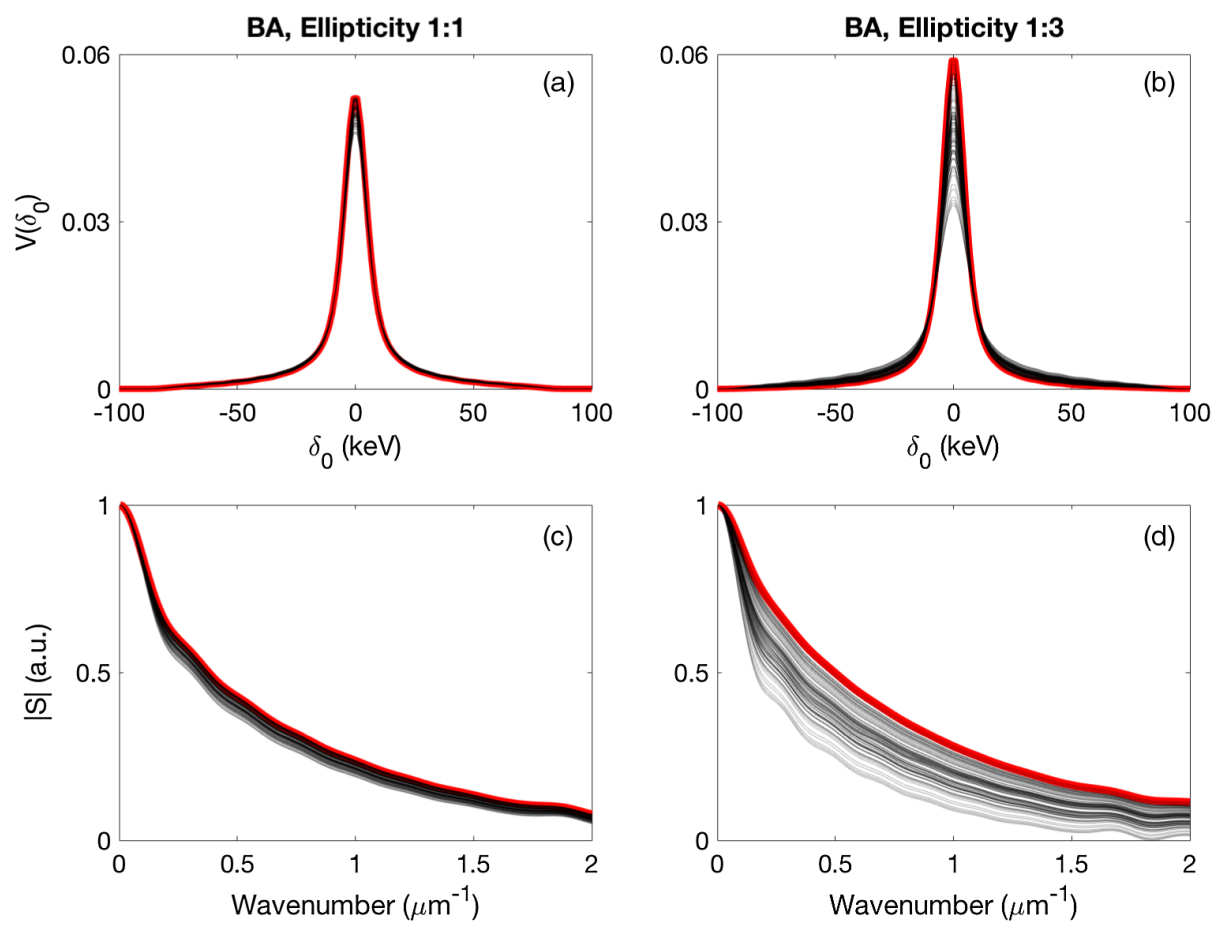

FIG. 7. Energy redistributions and suppression factor plots for BA. Energy spreads are optimized for rms energy spread.

$20 \mathrm{keV}$, while $70 \mathrm{MW}$ induces a FWHM energy spread of the same magnitude. We will use these parameters in the forthcoming analysis.

Significantly higher peak power levels are needed for the BA distribution in comparison to the Gaussian case. These high power requirements are the result of a large number of beamlets, many of which rarely interact with the e-beam. Some significant benefits of the BA design lie in the ability to individually control beamlets through iterative software, combining information about e-beam location and shape with FEL performance to tune the BA profile. This could minimize power consumption while maximizing MBI suppression.

\section{B. MBI suppression of BA}

Having found optimal parameters for our given e-beam size, we incorporate transverse offset jitter with a transversely circular e-beam. The introduced jitter is the same as used in previous simulations. The overlay of energy distributions, Fig. 7, generated with these parameters indicates that the energy distribution remains almost entirely constant, with a mean spread of around $20 \mathrm{keV}$ rms and a standard deviation of $0.6 \mathrm{keV}$. When the e-beam distribution is overlayed over a random portion of the BA, the induced energy shift remains approximately constant because the number of beamlets interacting with the bunch is the same. In relation to both Gaussian and LG01 beams (Fig. 3), the BA is able to maintain heating comparatively insensitive to jitter and thus maintains similar energy spreads and smooth distributions over a large range of offsets.

Figure 7 shows the distribution overlay, which remains smooth, with an average energy width close to $20 \mathrm{keV}$ rms. Though there is some increased variability in the energy distributions, this variation is not especially significant. In comparison to Gaussian and LG01 beams, the variation in energy distributions when using ideal and realistic e-beams is minimal: not only does the energy distribution change much less significantly, the curves have predictable, smooth shapes.

Though the BA energy distributions remain very stable, their shape is more Lorentzian than Gaussian. The

TABLE III. Energy spread and $I$ values for BA for both rms and FWHM optimization.

\begin{tabular}{lcccccc}
\hline \hline Laser profile & Energy spread & e-beam ellipticity & $P_{L}(\mathrm{MW})$ & $\delta_{E}(\mathrm{keV})$ & $I\left(\mathrm{~m}^{-1}\right)$ & $\mathrm{stdev}$ of $I\left(\mathrm{~m}^{-1}\right)$ \\
\hline BA & rms & $1: 1$ & 12 & $20.0 \pm 0.6$ & $1.3 \times 10^{5}$ & $0.2 \times 10^{5}$ \\
& & $1: 3$ & 15 & $22 \pm 2.5$ & $1.1 \times 10^{5}$ & $0.5 \times 10^{5}$ \\
& FWHM & $1: 1$ & 70 & $19.5 \pm 0.8$ & $1.7 \times 10^{4}$ & $0.2 \times 10^{4}$ \\
& & $1: 3$ & 100 & $21 \pm 3$ & $1.4 \times 10^{4}$ & $0.6 \times 10^{4}$ \\
\hline \hline
\end{tabular}


Lorentzian shape means that optimizing energy distributions for using FWHM rather than rms results in more consistent and effective heating. Quantified results are summarized in Table III. We find that for the ideal e-beam, $I_{\mathrm{FWHM}}=$ $1.7 \times 10^{4} \mathrm{~m}^{-1}$ with a standard deviation of $0.2 \times 10^{4} \mathrm{~m}^{-1}$. For the elliptical e-beam, $I_{\text {FWHM }}=1.4 \times 10^{4} \mathrm{~m}^{-1}$ with a standard deviation of $0.6 \times 10^{4} \mathrm{~m}^{-1}$. Optimizing for rms energy spread results in less suppression, with $I_{\mathrm{rms}}=1.3 \times$ $10^{5} \mathrm{~m}^{-1}$ and a standard deviation of $0.2 \times 10^{5} \mathrm{~m}^{-1}$. Though the suppression values are worse than the Gaussian case, the variance is significantly decreased, indicating the consistency of heating. The profile performs comparably worse when using the rms measurement, as changes in the broad sidebands of the Lorentzian-like energy distributions make rms energy spread vary significantly.

The results of the study indicate that heating is largely consistent, independent of e-beam shape and offset. Though this bunching gain $I$ is not as low as the optimal LG01 cases, the consistency of heating curves makes the BA a valid alternative. This makes this profile a candidate for systems in which the e-beam profile is inconsistent and widely varying. Beyond these benefits, the fact that laser parameters do not need to be adjusted significantly for vastly different e-beam shapes showcases the BA design as a highly practical and versatile approach.

\section{CONCLUSION}

Effective MBI suppression continues to be an unresolved challenge for optimal FEL performance. At LCLS, advanced operational modes such as harmonic lasing and self-seeded soft $\mathrm{x}$-ray emission already suffer from this shortcoming [12-15], and MBI suppression becomes particularly critical as x-ray FELs gear up to push the technological boundaries towards high repetition rate, attosecond duration, and higher photon energies at existing or prospective facilities such as Eu-XFEL and LCLS-II/HE.

In this work we have shown that the LG01 laser profile suppresses MBI far more effectively than the Gaussian laser profile, both with and without consideration of practical effects. The profile's performance is remarkably independent on a small eccentricity in e-beam transverse profile. Its performance can be improved with circular e-beam profiles and increased stabilization to the laser and e-beam. As a secondary benefit, the LG01 transverse intensity distribution can be generated relatively simply from a Gaussian distribution through holographic methods [19] or employing phase plates [20], among others. Thus upgrading existing LH systems to LG01 modes is minimally invasive. A consideration of this enhancement is its increased power consumption in comparison to the Gaussian case.

We also describe a second alternative, the Gaussian BA, which does not yield the level of MBI suppression that the LG01 mode does, but is largely insensitive to instabilities between laser and e-beam, and e-beam eccentricity. This design is motivated by a mathematically ideal BA that has been suggested in previous work [16] and represents an initial investigation into a feasible and realizable $\mathrm{BA}$ distribution. We find that the Gaussian BA maintains virtually identical energy distributions with tunable energy spreads regardless of e-beam size, shape, or offset. This profile is a good candidate for systems in which e-beam shape is largely variable shot to shot. As stated previously, performance of this intensity distribution could be improved significantly through the incorporation of non-Gaussian beamlets, combining the stability of the BA much better suppression. The BA design could also be improved through the control of individual beamlet sizes and powers to optimize heating of nonuniform or rapidly varying e-beams. The use of individual beamlets also presents the possibility of incorporating adaptive optics and algorithms to adapt to and predict e-beam shapes to tailor the e-beam energy distribution, minimizing power consumption while maximizing MBI suppression.

While energy distributions, suppression factor, and bunching gain are relevant and useful metrics for studying MBI suppression, a full analysis of FEL performance and LH effectiveness requires propagation of energy distributions from this study down the FEL. These shortcomings are exemplified by the contrast between FWHM and rms definitions. The proper uncorrelated energy spread induced by the LH can Landau damp the microbunching instability in the bunch compressors, altering the shape of the energy and electron distributions before the undulator. This as well as more advanced LH profiles based on the BA distribution are being investigated as part of ongoing work.

\section{ACKNOWLEDGMENTS}

This work was supported in part by the U.S. Department of Energy, Laboratory Directed Research and Development program at SLAC National Accelerator Laboratory, under Contract No. DE-AC02-76SF00515, and by the U.S. Department of Energy, Office of Science, Office of Workforce Development for Teachers and Scientists (WDTS) under the Science Undergraduate Laboratory Internship (SULI) program.

[1] Z. Huang et al., Measurements of the linac coherent light source laser heater and its impact on the x-ray free electron laser performance, Phys. Rev. ST Accel. Beams 13, 020703 (2010).

[2] M. Pedrozzi et al., The laser heater system of swissfel, in Proceedings of FEL2014, Basel, Switzerland (JACoW, Lausanne, Switzerland, 2014).

[3] P. Emma et al., First lasing and operation of an ångstromwavelength free-electron laser, Nat. Photonics 4, 641 (2010).

[4] C. Bostedt, S. Boutet, D. M. Fritz, Z. Huang, H. J. Lee, H. T. Lemke, A. Robert, W. F. Schlotter, J. J. Turner, and 
G. J. Williams, Linac Coherent Light Source: The first five years, Rev. Mod. Phys. 88, 015007 (2016).

[5] F. Lehmkuehler et al., Sequential single shot x-ray photon correlation spectroscopy at the SACLA free electron laser, Sci. Rep. 4, 5234 (2014).

[6] T. Ishikawa et al., A compact x-ray free-electron laser emitting in the sub-ångström region, Nat. Photonics 8, 540 (2012).

[7] S. Spampinati et al., Laser heater commissioning at an externally seeded free-electron laser, Phys. Rev. ST Accel. Beams 17, 120705 (2014).

[8] D. Ratner, C. Behrens, Y. Ding, Z. Huang, A. Marinelli, T. Maxwell, and F. Zhou, Time-resolved imaging of the microbunching instability and energy spread at the Linac Coherent Light Source, Phys. Rev. ST Accel. Beams 18, 030704 (2015).

[9] J. Qiang, R. D. Ryne, M. Venturini, A. A. Zholents, and I. V. Pogorelov, High resolution simulation of beam dynamics in electron linacs for x-ray free electron lasers, Phys. Rev. ST Accel. Beams 12, 100702 (2009).

[10] Z. Zhang, R. Lindberg, W. M. Fawley, Z. Huang, J. Krzywinski, A. Lutman, G. Marcus, and A. Marinelli, Microbunching-instability-induced sidebands in a seeded free-electron laser, Phys. Rev. ST Accel. Beams 19, 050701 (2016).

[11] Z. Huang, M. Borland, P. Emma, J. Wu, C. Limborg, G. Stupakov, and J. Welch, Suppression of microbunching instability in the linac coherent light source, Phys. Rev. ST Accel. Beams 7, 074401 (2004).

[12] E. Allaria et al., Two-stage seeded soft-X-ray free-electron laser, Nat. Photonics 7, 913 (2013).

[13] J. Amman et al., Demonstration of self-seeding in a hard-X-ray free-electron laser, Nat. Photonics 6, 693 (2012).

[14] D. Ratner et al., Experimental Demonstration of a Soft X-Ray Self-Seeded Free-Electron Laser, Phys. Rev. Lett. 114, 054801 (2015).

[15] Z. T. Zhao et al., First lasing of an echo-enabled harmonic generation free-electron laser, Nat. Photonics 6, 360 (2012).

[16] S. Li et al., in Proceedings of FEL2015, Daejeon, Korea.

[17] A. Marinelli and J. Rosenzweig, Microscopic kinetic analysis of space-charge induced optical microbunching in a relativistic electron beam, Phys. Rev. ST Accel. Beams 13, 110703 (2010).

[18] http://physics-elog/lclselog/index.jsp.

[19] J. Arlt, K. Dholakia, L. Allen, and M. J. Padgett, The production of multiringed LaguerreGaussian modes by computer-generated holograms, J. Mod. Opt. 45, 1231 (1998).

[20] L. Allen, M. W. Beijersbergen, R. J. C. Spreeuw, and J. P. Woerdman, Orbital angular momentum of light and the transformation of Laguerre-Gaussian laser modes, Phys. Rev. A 45, 8185 (1992). 